\title{
LA COMPETENCIA DEL TRADUCTOR QUE NO "TRADUCE": EL TRADUCTOR EN ÁMBITOS DE INTERNACIONALIZACIÓN EMPRESARIAL
}

\author{
Marián Morón Martín \\ mmoron@upo.es \\ Universidad Pablo de Olavide \\ Ana Medina Reguera \\ anamedina@upo.es \\ Universidad Pablo de Olavide
}

\section{Resumen}

En este trabajo se aborda la inserción profesional, la formación y el currículo académico en los estudios de Traducción e Interpretación (TeI) como objetos de investigación empírica. Más específicamente, nos centraremos en la observación de perfiles de egresados en TeI que están trabajando en ámbitos de internacionalización empresarial (en empresas que desean exportar bienes y servicios o invertir en el exterior). Con especial atención a los puestos desempeñados, publicados en la red social profesional LinkedIn, y su autopercepción identitaria, nuestro objetivo es, por un lado, conocer más profundamente la realidad de estos perfiles afines (Morón 2010, Calvo 2010) y, por otro, aproximarnos a la autopercepción de los sujetos como profesionales de este ámbito dada su formación específica en TeI. A través de este estudio, las autoras pretenden reflexionar sobre el concepto de competencia traductora (CT) en su aplicación didáctica para responder más acertadamente a las demandas del mercado.

\begin{abstract}
"Translation competences of translators who do not 'translate': Translators in professional fields of business internationalization"

This paper deals with employability, training, and the academic curriculum in the Bachelor's Degree of Translation and Interpreting (TI) as empirical research objects. More specifically, it focuses on the observation of the professional profile of TI graduates currently working in the field of business internationalization (in companies
\end{abstract}


that wish to export goods and services or make investments abroad). Paying special attention to the positions held, as published in the professional social network LinkedIn, and their identity self-perception, this piece of work aims, on the one hand, to gain deeper knowledge of translation-related professional profiles (Morón 2010, Calvo 2010) and, on the other, to get to know our subjects' self-perception on their professional profiles given their specific TI training. Thanks to this study, the authors aim to reflect on the translator competence (TC) concept from a pedagogical point of view, with the purpose of better responding to market needs.

Palabras clave: Inserción profesional. Competencia traductora. Traducción para el comercio internacional. Comercio exterior. Internacionalización de empresas.

Keywords: Employability. Translation competence. Translation and foreign trade. International commerce. Companies' internationalization.

Manuscript received on July 6, 2015 and accepted for publication on October 16, 2015. 


\section{Introducción}

La actividad internacional de las empresas es una de las actividades que más práctica traslativa supone para las agencias o empresas proveedoras de servicios de traducción. No en vano, es frecuente leer como parte fundamental de los servicios ofertados por estas agencias reclamos tales como "traducción para los negocios", "soluciones integrales para la empresa exportadora" o "traducción de documentación comercial y de exportación". ${ }^{1}$ Cubrir las demandas de transferencia lingüística de las empresas que se deciden a introducir sus productos en otros países o mercados con una lengua diferente a la española no es, por tanto, una invención del siglo XXI. Otro de los indicadores sobre la tradición de la traducción comercial en España es la frecuente -o incluso obligatoria- inclusión de textos vinculados a este sector en la didáctica de la traducción. Estos textos han sido incorporados a la formación de traductores desde los inicios de las licenciaturas de Traducción e Interpretación (TeI) en España, y no resulta extraño observar en programas antiguos o actuales la presencia de textos de correspondencia comercial, contratos internacionales de compraventa, formularios para el transporte, documentos sobre el seguro o el pago de las mercancías, etc. Todos forman parte de la formación básica de los futuros traductores, y la bibliografía sobre didáctica de la traducción comercial publicada en España, aunque no es muy profusa, ha dado también buena cuenta de ello (Socorro 2008, 2012; Mayoral 2006; Suau 2010; Medina 2007; Román 2008, 2012; Aguayo y Morón 2013, entre otros).

También en los ámbitos de postgrado han surgido programas más específicos, con especial atención a la traducción económica, financiera o institucional como campos de especialización para el traductor. Y, en algunas universidades privadas españolas, incluso se han inaugurado algunos Dobles Grados de Relaciones Internacionales y TeI.

Aun cuando la traducción para el comercio internacional no sea una novedad de los últimos años, sí debemos afirmar que ha crecido paulatinamente

1. Expresiones citadas de las respectivas páginas de inicio de las empresas molmola.com, idisc.com y planetlingua.com, respectivamente. [Último acceso: 25/2/2015] 
al tiempo que las empresas españolas han ido internacionalizándose también cada vez más. A diferencia de otros países con larga trayectoria exportadora, como Alemania o Francia, España ha experimentado durante el siglo XX un déficit estructural en su balanza comercial (Navarro 2007: 323) y un tejido empresarial local, familiar y poco industrializado (García y Manera 2006). En España, el 99,88\% del tejido empresarial está constituido por pymes (pequeñas y medianas empresas cuya plantilla oscila entre 0 y 249 empleados), de las cuales el 95,5\% son microempresas (entre 0 y 9 empleados). ${ }^{2}$ Para mejorar esta situación, han proliferado en los últimos años programas de apoyo y subvenciones que, unidos a la fuerte crisis económica del país, han empujado a las empresas a buscar clientes fuera de las fronteras para exportar los productos y servicios que no tenían cabida en el mercado nacional. Pues bien, de forma natural y lógica, el aumento de la internacionalización trae consigo el aumento de la Traducción para el Comercio Exterior, y hemos tratado de analizar el fenómeno en trabajos anteriores desde perspectivas variadas (Aguayo y Ramírez 2011; Morón 2012, 2015; Morón y Aguayo 2013; Medina y Álvarez 2014; Medina y Ramírez 2015a, 2015b; Medina 2015; Álvarez 2015; proyecto COMINTRAD). ${ }^{3}$

En este contexto general, queremos analizar los resultados de nuestras observaciones regulares y controladas sobre cómo los entornos de trabajo de los titulados en TeI van evolucionando, ante su paulatina incorporación al sector empresarial, ${ }^{4}$ y no precisamente bajo la denominación del puesto laboral de "traductor", sino usando otras denominaciones que parecían reservadas a los egresados de otras titulaciones.

Tal y como refleja el reciente trabajo de Álvarez (2015), los egresados en TeI que acceden a estos puestos en empresas internacionales afirman traducir, pero traducir poco (correos electrónicos, folletos y catálogos y, menos frecuentemente, contratos o estudios de mercado), lo que casa con las observaciones realizadas en nuestras investigaciones previas (Álvarez 2015). De hecho, si se

2. Retrato de las PYME 2014. Disponible en: <http://www.ipyme.org/Publicaciones/Retrato_ PYME_2014.pdf> [Último acceso: 25/2/2015]

3. Proyecto I+D+i del Ministerio de Economía y Competitividad de la convocatoria 20132016 del Programa Estatal de Investigación, Desarrollo e Innovación orientada a los Retos de la Sociedad: "Comercio Internacional y Traducción: Localización de webs corporativas y E-Marketing Multilingüe para fomentar la internacionalización de las pymes españolas (proyecto COMINTRAD, Ref. FFI2013-44550-R)", bajo la dirección de Ana Medina Reguera, de la Universidad Pablo de Olavide de Sevilla.

4. Debemos puntualizar que nos referimos en todo momento a la incorporación de egresados en empresas que no pertenecen a la industria de la lengua, es decir, empresas de traducción o gestión lingüística, editoriales, instituciones privadas de enseñanza, etc. 
analizan con mayor detenimiento aquellas tareas marcadas lingüísticamente asociadas a entornos de internacionalización empresarial, detectamos que, en lugar de traducción o interpretación "pura" o "clásica", se realizan tareas de mediación lingüística o comunicación multilingüe, en muchos casos combinadas con traducciones. Tal y como defendieran Mayoral (2006) o Socorro (2012), la traducción en estos entornos empresariales está más cercana a la redacción o producción lingüística que al ejercicio de la TeI.

Por otra parte, estos egresados suelen trabajar con textos de naturaleza varia, pero también han de realizar actividades muy diversas: lectura, traducción a vista, traducción, traducción adaptada, localización, etc. Y frecuentemente no solo en un par de lenguas, sino en varias. Resulta pues evidente que el traductor integrado en estos entornos afines no hace el mismo uso de sus competencias que en entornos de traducción "clásicos". En consecuencia, parece que su dominio de las nuevas herramientas tecnológicas de traducción o de gestión terminológica queda relegado a un segundo término, mientras que sus competencias interculturales, interpersonales, temáticas o estratégicas tienden a adquirir un mayor protagonismo.

Con un enfoque más cualitativo y centrado en el individuo, este trabajo pretende arrojar algo de luz sobre los caminos que estas salidas e itinerarios profesionales suponen para la formación específica en TeI y contribuir a lograr una mejor definición de la propia profesión del traductor, alineándose con otros trabajos precedentes como Kuznik (2010), Torres (2012) o Pym, Grin, Sfreddo \& Chan (2013).

\section{La oferta y la demanda de traducción para la empresa exportadora}

Los cambios que ha producido la web en los patrones de comunicación humana son indiscutibles. La posibilidad de comunicarse virtualmente o de acceder remotamente a archivos de hipertexto ha cambiado radicalmente la forma de interactuar y de relacionarse en todo el mundo. Para las empresas, la web significa que pueden pasar de ser locales a ser globales, dándole una nueva dimensión a la globalización económica y situándolas en lo que ha venido denominándose la nueva economía o la sociedad de la información y el conocimiento, ambos conceptos directamente ligados al auge de las empresas virtuales o punto-com. ${ }^{5}$ Las pymes pueden presentarse al mundo con sitios o blogs, pueden

5. El término nueva economía está directamente ligado al fenómeno de la globalización económica y fue acuñado por el economista Brian Arthur para describir el auge de la economía basada en el conocimiento y en las tecnologías de la información y comunicación (Friedman 2006). 
publicitarse y obtener beneficios por su publicidad en Internet, pueden vender de forma electrónica o buscar clientes de forma masiva. Sin embargo, en esta revolución tecnológica, la barrera del idioma sigue presente (ELAN 2006, Rico, s.d.). Pese a los avances en traducción automática, esta no ofrece todavía resultados lo suficientemente válidos como para sustituir la traducción humana. Las pymes españolas se enfrentan a retos en materia de nuevas tecnologías, desarrollo de negocio y, también, comunicación multilingüe. No en vano, algunos informes han apuntado las dificultades que conlleva la internacionalización en España respecto a otros países europeos, tanto en la desventaja de la falta de conocimiento de idiomas, como en el lento avance de la sociedad digital (Comisión Europea 2005; CIS 2014; Agenda Digital para España (MINETUR 2013) y Agenda Digital para Europa (Comisión Europea 2013).

Por su parte, el mercado de la TeI también ha evolucionado hacia nuevas realidades que hacen muy difícil establecer límites interdisciplinares. Internet ha propiciado un sustancial avance en la forma de hacer y estudiar la TeI, en tanto que han ido surgiendo nuevas realidades y nuevas demandas para estos profesionales. La necesidad de estar presentes en Internet ha llevado a las empresas a un mundo donde la transferencia lingüística tiene un valor añadido. Si antes ya se presuponía que la empresa interesada en acceder al mercado internacional iba a necesitar de un cierto grado de intercambio lingüístico, más aún ocurre ahora con el acceso al mercado virtual, donde el inglés sigue siendo la lengua vehicular de los negocios internacionales y la que mayor presencia tiene en el mundo digital. Los informes de CSA (Common Sense Advisory) señalan cómo la internacionalización empresarial resulta uno de los pilares de la actividad traductora actual, con demandas centrales en localización de software y de aplicaciones móviles, localización de sitios web (Jiménez 2013, Alonso y De la Cova 2013) y presencia multilingüe en las redes sociales. A su vez, parece que la actividad de "no traducir" se ha convertido, paradójicamente, en parte del quehacer de muchos traductores. El auge de la post-edición (Romaine y Richardson 2009), de la traducción intralingüística intersemiótica y multimodal (subtitulación o audiodescripción), de la producción y gestión multilingüe o la creciente visibilidad de la traducción publicitaria o creativa (o "transcreación" como se la conoce

6. Sales (2004) analiza el concepto desde la perspectiva de la traducción literaria y califica la transcreación como una traducción creativa, transgresora, estética y artística. Rike (2013: 73), más cercana al enfoque de nuestro estudio (centrada en la traducción de sitios webs corporativos), destaca la cercanía de esta actividad con la localización (más centrada en la traducción de software, manuales de instrucciones o de usuario), aunque la transcreación supone un enfoque diferente y más acusado de creatividad. 
recientemente por agencias y empresas del sector) son precisamente ejemplos de cómo la globalización e internacionalización de los mercados deriva en nuevas realidades a las que el traductor está haciendo frente. En este contexto, el traductor está mostrando como profesional su virtual capacidad de inserción en otros sectores vinculados a la actividad empresarial internacional, tales como el comercio internacional, el protocolo, las relaciones institucionales o diplomáticas, la mediación intercultural, la publicidad, la informática o el marketing electrónico. En todos ellos la comunicación multilingüe, la conciencia de diversidad cultural, las nuevas tecnologías y los conocimientos sobre las dinámicas de las relaciones internacionales resultan fundamentales, salvando las diferencias entre sectores.

El mercado laboral, por tanto, ha comenzado a buscar profesionales cada vez más multilingües, y además, cada vez más polivalentes, flexibles, que puedan afrontar la volatilidad de los mercados y los cambios vertiginosos de las formas de trabajo con las nuevas tecnologías. Se valoran en gran medida las competencias transversales o profesionales que puedan favorecer esta flexibilidad: las competencias comunicativas (hablar idiomas, comunicar de forma efectiva y estratégica), las competencias culturales (saber desenvolverse en distintos entornos), las competencias tecnológicas (dominio de las nuevas tecnologías), entre otras (ANECA 2004: 31). De igual modo, las universidades, quizás movidas por las dinámicas del mercado, o influidas por la reforma del Espacio Europeo de Educación Superior (con su apuesta por la movilidad y la flexibilidad), o por una combinación de ambas, también parece que están reaccionando ante esta demanda de mayor formación interdisciplinar.

Por otro lado, los pocos datos conocidos referentes a la empleabilidad de los titulados en TeI ya han revelado que el comercio internacional es uno de los principales nichos de empleo. Según el Libro Blanco del Grado en TeI de la ANECA (2004), el comercio internacional se situó como el tercer sector de empleo en número de egresados empleados (11\%), datos compartidos con los reflejados en estudios parciales de otras universidades (Calvo 2006). Los datos de Torres (2012: 789), por su parte, indican que los "administrative jobs in companies" representan hasta el $46 \%$ en la Universidad Autónoma de Barcelona entre 1999 y 2003. También en el estudio de Morón (2010), el comercio internacional se erigía en el sector dilecto de los egresados de Traducción y Lenguas, donde casi el 19\% de los sujetos encuestados (de un total de 85 graduados) se ubicaba en este sector, fuera en combinación o no con el ejercicio de la traducción (frente al 14\% que se dedicaba exclusivamente a la traducción). De forma similar, los titulados en Filología o Traducción ocupaban el quinto puesto en número de becarios de internacionalización 
empresarial del programa de becas del ICEX de 2008, y suponían el 42\% de los becarios del programa de Informática, lo que los situaba en el primer puesto de las titulaciones que lograron dichas becas, incluso por delante de los egresados en Ingenierías Informáticas (Morón 2012).

Con todo lo anterior como punto de partida, este trabajo se plantea reflexionar sobre aquellos componentes de la competencia traductora (CT) que pueden verse reforzados a la hora de atender estas orientaciones profesionales relacionadas con la comunicación internacional, la interculturalidad empresarial y las nuevas tecnologías, y responder, más adecuadamente, a las demandas actuales de las empresas españolas que son (o desean ser) parte del mercado global. Por consiguiente, en nuestra investigación, aprovechamos los mensajes y la retroalimentación que se desprende del propio mercado: hemos formado a egresados que, con su empleabilidad, demuestran ser competentes para atender las necesidades de las empresas internacionales y, a su vez, estos perfiles observados nos sirven para verificar, completar y redefinir la CT como elemento rector de la formación de estos futuros profesionales. Por último, si los docentes llegamos a conocer estos casos de éxito de nuestros antiguos alumnos, tendremos ejemplos reales con los que motivar a nuestros futuros alumnos. Afrontaremos, por tanto, la paradoja de la formación especializada en la que parecen sustentarse nuestros Grados y la mayor parte de la oferta formativa nacional, frente a la diversidad o polivalencia del perfil del egresado en el mercado laboral (Mayoral 2004, Aneca 2004, Morón 2012).

\section{La competencia traductora hoy: un balance}

El concepto de competencia traductora ha suscitado un interesante y prolífico debate en Traductología, especialmente en los últimos años, animado por el propio devenir del modelo educativo donde la competencia se ha convertido en el pilar sobre el que construir los programas de formación. Los modelos de competencia iniciales parecen surgir como elemento diferenciador del "saber traducir" frente al "saber hablar idiomas" (Wilss 1976, Kiraly 1995). La investigación en torno al concepto CT ha sido fructífera, a la par que cuestionada (Mayoral 2001, Pym 2003) y ha reflejado, en sus aproximaciones, los propios debates epistemológicos e ideológicos (Kearns 2012) de la disciplina. Parece lógico que a definiciones diferentes para traducir y traducción, le correspondan diferentes modelos de CT. Estudios como los de Wilss (1976), Delisle (1980), House (1981), Nord (1991) o Gile (1995) han contribuido a construir una pedagogía más sólida de la traducción en la que construir los modelos competenciales actuales, sin olvidar la influencia ejercida por el cambio en 
el concepto de texto, que ha sido profundo debido al desarrollo de las nuevas tecnologías (Jiménez 2013: 43-49).

Algunos modelos y formas de aproximarse a la CT parten de la traducción como conocimiento experto, que puede estudiarse, objetivarse y analizarse cognitivamente (Gile 1995; PACTE 2009, 2014, entre otros) y se centran en el proceso de la traducción profesional. Autores como Kiraly (1995) añaden un enfoque psicolingüístico y se centran también en los procesos mentales (intuitivos y controlados) que ocurren durante la actividad traductora. La prolífica investigación del grupo PACTE, cuyo trabajo se centra fundamentalmente en el estudio empírico-experimental de los procesos cognitivos subyacentes a la adquisición, desarrollo y evaluación de la CT, ha ido abordando progresivamente las subcompetencias de la CT desde finales de los años noventa. ${ }^{7}$ Por otro lado, otros enfoques, como el de Kelly (2002), toman su modelo de CT como guía en el proceso de enseñanza-aprendizaje, desde un paradigma crítico y no positivista.

Por consiguiente, existen múltiples enfoques desde los que afrontar la investigación en torno a la CT e igualmente resultan múltiples los modelos descritos (más sencillos o más complejos en cuanto al número de componentes o subcompetencias) y su diversa contextualización. Morón (2010), desde una perspectiva didáctica, se hace eco de algunos de los debates recurrentes a este respecto:

- La indefinición del constructo o de ciertos componentes o subcompetencias de la CT.

- La definición meronímica y la contraposición de modelos minimalistas y modelos multicomponenciales.

- El enfoque profesional y la inclusión de las competencias transversales en los modelos de CT.

- El valor de la CT como modelo para el diseño de propuestas didácticas concretas.

A pesar de esta diversidad, que refleja la riqueza de la investigación en nuestro ámbito, la naturaleza de las propuestas existentes ha tendido a ser bastante homogénea (Calvo 2010) en tanto que los modelos resultantes han mostrado la especificidad de la labor traductora, destacando el papel de las competencias comunicativas, culturales y profesionales (instrumentales, estratégicas,

7. Sus distintos trabajos sobre la CT pueden consultarse desde la página web del grupo PACTE (Procés d'Adquisició de la Competència Traductora i Avaluació), en: <http:// grupsderecerca.uab.cat/pacte/content/publicacions $>$. 
interpersonales y psicológicas) (PACTE 2014). Es quizás en este último bloque donde se aprecian mayores avances. Los modelos más recientes tienden a reflejar en mayor medida las competencias instrumentales y profesionales, fruto de la importancia de las nuevas tecnologías en la práctica traslativa. En esto, el peso de las competencias transversales y la importancia de la adquisición gradual de la competencia (Robinson 1997, 2003; Mayoral 2001; Kiraly 2013) hace que competencias genéricas como la resolución de problemas, la toma de decisiones, la capacidad de adaptación, de trabajo en equipo y la gestión del tiempo, entre otras, adquieran un valor añadido a la luz de los avances de la propia profesión y los nuevos métodos de trabajo (en red, crowdsourcing, deslocalización de equipos, volatilidad del mercado, etc.). Hasta el punto de que PACTE (2014: 86) llega a considerar que las subcompetencias más específicas del traductor profesional (en oposición a cualquier hablante bilingüe) son la competencia estratégica (con un papel central en su modelo), la instrumental y los conocimientos sobre la Traducción.

En este trabajo, las autoras adoptarán el enfoque didáctico del concepto de CT (Kelly 2002), y se plantearán una adaptación del modelo de CT que dé una mejor respuesta al egresado que pretenda adentrarse en entornos empresariales. Este énfasis en la empleabilidad ha sido uno de los aspectos destacados en la elaboración de los nuevos planes de estudio en el EEES según las directrices del Plan Bolonia, y varios autores han defendido la empleabilidad y la formación para el mercado a lo largo de los últimos años (Gouadec 2007, Schäffner 2012, entre otros). Este hecho no significa que los contenidos y objetivos curriculares deban quedar sujetos a los vaivenes de los mercados (véase al respecto las críticas de Pym 2003, Kearns 2012, Ulrych 2005); en nuestra propuesta, por el contrario, valoramos y deseamos hacer valer aquellas competencias transversales, establecidas en los planes y guías docentes, que están dando su fruto en entornos empresariales entre los egresados españoles. Así describían el pronóstico Chouc y Calvo (2011: 72): "[E]mployability is not only about responding to short-term market needs (a quantitative perspective based on the specialised profiles most in demand at a given time), but rather about generating competent, active social agents that are able to react to any given context (qualitative view)".

\section{Método de la investigación}

Para llevar a cabo este estudio hemos seguido un método de investigación centrado en el sujeto y más concretamente en sus manifestaciones textuales. Adoptaremos pues un método de investigación y análisis eminentemente cualitativo (Cook y Reichardt 1979, Cohen y Manion 1980), de corte descriptivo 
y exploratorio (Hernández, Fernández Collado \& Baptista Lucio 2003). Según este último, estos estudios pretenden "especificar las propiedades, las características y los perfiles importantes de personas, grupos, comunidades o cualquier otro fenómeno que se someta a un análisis (Danhke 1989), así como medir, evaluar o recoger datos sobre diversos aspectos, dimensiones o componentes del objeto de investigación". Colás (1998: 177), claramente en línea con los objetivos que motivan nuestra investigación, añade que los estudios cualitativos ayudan a proporcionar datos y hechos, identificar problemas, fenómenos relevantes, dar pautas para la configuración de teorías, plantear hipótesis, identificar áreas de estudio que no pueden ser tratadas experimentalmente, así como realizar comparaciones y evaluaciones, planificar futuros cambios y toma de decisiones. En nuestro caso, adoptaremos la técnica del análisis de contenido (cuantitativa), gracias a la que inferiremos (enfoque cualitativo) aquello que motiva las manifestaciones de los sujetos (López 2002). Esta técnica consiste en el estudio sistemático y objetivo de las comunicaciones o discursos, según las variables que procedan en la investigación, que han de ser operacionalizadas por el investigador. López (2002: 168) sugiere que este método "no puede ser indistintamente aplicado a la educación en general, sino a los diversos aspectos y procesos en que se traduce el hecho educativo." El autor justifica la relevancia del método en Educación al ser "un instrumento de respuesta a esa curiosidad natural del hombre por descubrir la estructura interna de la información, bien en su composición, en su forma de organización o estructura, bien en su dinámica." (López 2002: 173). De esta forma, se pretende analizar cómo se manifiesta una determinada característica del sujeto (por ej., la motivación) o un determinado tema (por ej., gracias al análisis de frecuencias) en sus comunicaciones. En nuestro caso, como sugiere López (2002), se utilizará esta técnica para analizar las percepciones del sujeto respecto a su propia formación y desempeño profesional a través de sus publicaciones en los perfiles en la red social profesional LinkedIn, y durante las comunicaciones personales mantenidas con las investigadoras sobre su actual sector y puesto de trabajo.

LinkedIn (<www.linkedin.com>) es una red social profesional de gran éxito y fundada en 2003 en Estados Unidos. Actualmente cuenta con más de 364 millones de usuarios en más de 200 países y está disponible en 24 lenguas. ${ }^{8}$

Se realizará pues un estudio de corte intensivo (centrado en un número limitado de documentos) (López 2002: 172) basado en la deducción o

8. Según la información en <https://www.linkedin.com/company/linkedin> 
inferencia (Bardin 1986:7, cit. en López 2002: 173), donde analizaremos las características y contenido mismo del texto, en base a los siguiente datos de corte cuantitativo:

- Ítem 1. Presencia o no de la traducción y la interpretación en la descripción de los perfiles descritos: tareas, puestos, empresas, formación de posgrado.

- Ítem 2. Sector de la empresa en la que los sujetos ejercen su actividad profesional y zona o país a que pertenece dicha empresa.

- Ítem 3. Especialización en comercio internacional o exterior en la formación de posgrado, si procede.

Así, se realizará un análisis de la frecuencia de aparición de la traducción o la interpretación en dichos perfiles (ítem 1) y se tomará nota y categorizarán las respuestas recogidas en los ítems 2 y 3 , para hacer un balance exploratorio sobre la naturaleza de los perfiles descritos.

Pese a lo anterior, lejos de aproximaciones estrictamente cuantitativas al fenómeno de la inserción laboral en sectores afines a TeI,, este trabajo se fundamenta en una muestra cualitativamente significativa de veinte sujetos que cumplen con el perfil profesional objeto de estudio, con un doble objetivo: 1) conocer más sobre las particularidades de sus perfiles profesionales (tareas o encargos frecuentes, tipo de puesto, sector de empleo); 2) aproximarnos a su autopercepción como profesionales de este nuevo ámbito. Por ello, nos centraremos únicamente en aquellos sujetos, egresados en TeI por la Universidad Pablo de Olavide de Sevilla (UPO), que están en activo en el momento de realización del estudio y que ejercen su actividad en empresas no lingüísticas con actividad internacional. Teniendo en cuenta que el perfil de la red social se organiza mediante una distribución coincidente con la de un currículum profesional (con distintos campos con información personal, profesional, académica, etc.), hemos de señalar que de los veinte egresados, no todos han rellenado su perfil con el mismo grado de detalle, por lo que no podemos recoger información cualitativa de todos y cada uno de los sujetos en ciertos elementos.

Para dar respuesta a los objetivos específicos señalados anteriormente, el análisis se articulará según estos elementos: 
1. Denominación del puesto: se trata de un campo de autoedición en el que el sujeto incluye su cargo en la empresa en la que trabaja.

2. Tipo de empresa o corporación para la que trabajan: este dato nos resulta de utilidad para conocer aquellos sectores que demandan los servicios de nuestros egresados.

3. Funciones del puesto que los profesionales han redactado en su perfil: de nuevo, se trata de una campo autoeditado por los participantes del estudio en el describen aquellas competencias, tareas, actividades, roles, etc., que definen su actual puesto de trabajo. En este sentido, el análisis nos puede ayudar a conocer más sobre la actividad real profesional de estos egresados, así como discernir si realmente incorporan la traducción o la interpretación como actividades propias de su empleo;

4. Formación de postgrado acreditada en el apartado del perfil: puesto que se asume que el sector de la internacionalización empresarial resulta un campo afín a la Traducción, entendemos necesario, para conocer en mayor grado de profundidad el perfil del egresado, si este ha seguido alguna formación de especialización tras completar sus estudios de Grado, que pueda haber favorecido o condicionado su acceso a este sector.

Recabamos un total de veinte perfiles que se adecuaban a los requisitos de nuestra investigación. Es necesario mencionar que hemos respetado siempre el anonimato de los sujetos, quienes, si bien facilitan su perfil de forma abierta, lo hacen únicamente para aquellos usuarios de la red. Se incorpora además información personal facilitada por los sujetos, contactados personalmente por las investigadoras. En estas comunicaciones personales, se aborda el segundo objetivo de nuestro estudio, en el que se analiza la autopercepción de los sujetos consultados. El contacto surge al invitarles a unas jornadas de orientación laboral para futuros egresados; a pesar de ello, en las respuestas, observamos sus visiones y actitudes respecto a la vinculación de su perfil de egreso con su perfil profesional). Somos conscientes de las limitaciones de nuestro estudio, centrado en un número limitado de sujetos, pero entendemos que esta primera fase de aproximación al estudio, de corte cualitativo y con un enfoque deductivo, nos puede ser de gran utilidad a la hora de diseñar estudios de mayor envergadura, que puedan arrojar más luz sobre el objetivo último de la investigación: conocer, valorar y responder al perfil profesional del traductor que trabaja en entornos de internacionalización empresarial. 


\section{Resultados del análisis}

5.1. Objetivo 1: Particularidades de los perfiles profesionales de egresados en TeI en entornos empresariales de comercio internacional

A continuación presentamos los resultados del estudio. Nuestra intención es, recordemos, mostrar algunos ejemplos reales de trayectorias profesionales de antiguos alumnos de la UPO. Todos ellos terminaron sus estudios de Licenciatura en TeI entre los años 2009 y 2011. Con este dato, podemos advertir claramente su ágil incorporación al mundo laboral, por una parte, pero también, su acceso a un sector de especialización afín a la TeI, lo que nos lleva a reflexionar en el peso positivo que la formación recibida (lingüística, tecnológica, traslativa, intercultural) tiene en este sector afín.

En cuanto al primer criterio de análisis (denominación del puesto), la herramienta LinkedIn nos permite acceder a las denominaciones de sus puestos actuales. Reproducimos aquí el cargo ocupado tal cual lo ha redactado cada persona, si bien el hecho de que el nombre del puesto esté redactado en inglés no se debe a que la persona en cuestión esté trabajando fuera de España. Tampoco podemos verificar si el puesto acreditado es el mismo que aparece en su contrato de trabajo o si es una versión más "exótica", pero sí sabemos que es una denominación que ellos mismos han redactado y no el resultado de la elección de un menú desplegable o de una traducción automática:

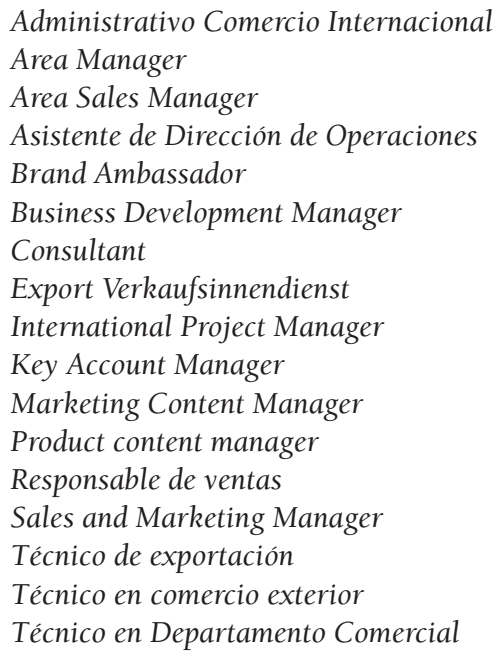

Tabla 1: Denominaciones de los puestos ocupados por egresados en la red profesional LinkedIn 
Ningún sujeto alude a la traducción o la interpretación en la denominación de sus puestos (Ítem 1).

En cuanto al segundo criterio (tipo de empresa para la que trabajan), hemos recogido empresas de todos los sectores económicos (agroalimentario, consumo, servicios e industria), siendo difícil establecer, en los casos recogidos, ningún tipo de afirmación representativa de la realidad de la muestra respecto al ítem 2 (sector empresarial). Se observa pues una gran dispersión en la empleabilidad de los sujetos consultados.

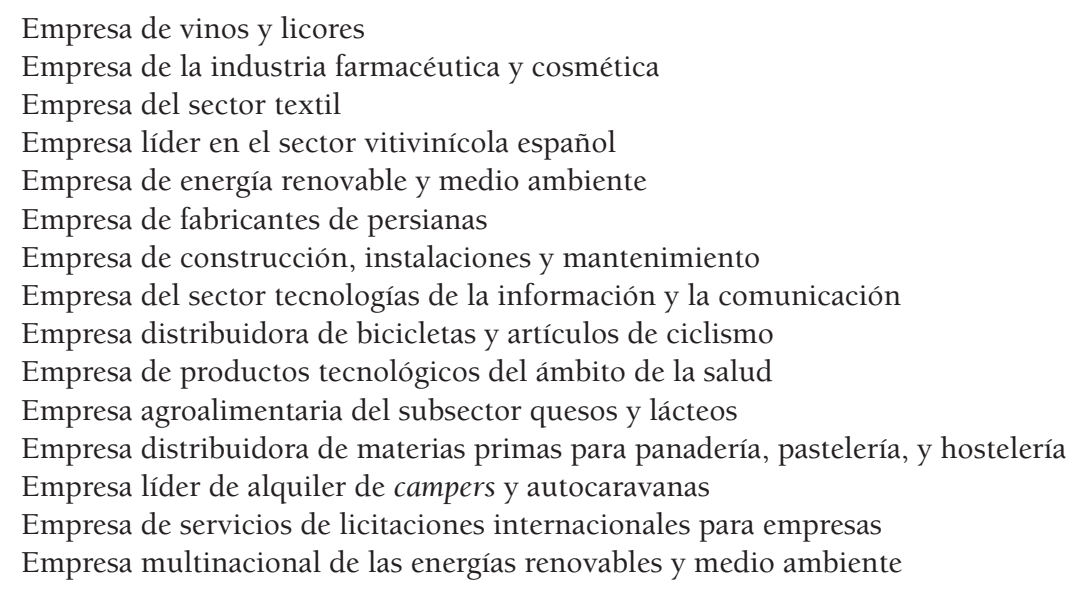

Tabla 2: Tipos de empresas en las que trabajan los egresados del perfil estudiado.

En 12 de los 20 casos se trata de empresas españolas o internacionales exportadoras de productos o servicios. Solo en 1 se trata de una empresa española importadora. El resto (8 empresas) son originarias de otros países europeos. El hecho de trabajar para una empresa española no significa que el egresado trabaje en España (cuatro egresados se encuentran en Asia y otros cuatro, en Francia, Alemania, Suiza y Colombia). De los 12 restantes, 7 trabajan en Andalucía y el resto se reparten en varias comunidades autónomas españolas.

Respecto de las funciones desempeñadas en estas empresas (tercer criterio), podemos tomar los datos desde la rúbrica "Extracto" (dentro del apartado "Trayectoria profesional y académica") o de la pestaña "Descripción" dentro del apartado "Puesto de trabajo". Aquí, el profesional puede redactar, según su propio criterio, las funciones principales de los puestos desempeñados. En algunas ocasiones se destaca la traducción de textos como funciones 
principales (egresados A y B); en otros, no se menciona la traducción en absoluto (egresados C y D):

Egresado A: "Captación de clientes nacionales e internacionales, gestión de cartera de clientes, organización de transporte de mercancías, traducción de documentos técnicos ING>ESP - ESP >ING y FR>ESP - ESP >FR, emisión y gestión de facturas."

Egresado B: "Support on managing demand, obtaining indicators, analyzing processes, seeking for improvement methodologies; - Support on preparing specicialized (sic.) guiding documentation and making translations (EN-ES-EN); - Support on the Business Relationship Management.; - Support on the preparation of IT Strategy documentation."

Egresado C: "Encargada de operaciones de importación: negociación y gestión logística, bancaria (medios de pago, seguros de cambio, financiaciones, etc.). Asistente en operaciones de exportación: encargada de procesos de registro de nuevos productos en países de Europa del Este y Oriente medio, cumplimentando y obteniendo la documentación requerida por los ministerios locales, además de realizar búsquedas de nuevas oportunidades de negocio y contactos con empresas en Suramérica."

Egresado D: "Con la oportunidad de abrir personalmente el departamento de exportaciones y reportando directamente al gerente de la empresa, mis principales funciones son: - Análisis de los mercados objetivo para la internacionalización de la empresa, colaborando en la decisión de los países que abordar y la forma de entrada; - Búsqueda de oportunidades de negocio en cada uno de los mercados, estudio de patrones de compra locales y de la competencia directa a nivel nacional e internacional; - Preparación de agendas comerciales y ferias internacionales; - Preparación de ofertas públicas (licitaciones) y privadas: documentación técnica y administrativa, preparación y envío de muestras, aclaración de dudas y supervisión de precios/márgenes; - Asegurar el correcto desarrollo integral de las operaciones internacionales: trato directo con clientes e intermediarios, gestión de proveedores nacionales/internacionales, orden de fabricación, control de stock, seguimiento y reclamación de cobros/pagos, resolución de incidencias; - Gestión de operaciones logísticas internacionales: gestión de transportistas y consolidadores, solicitudes de cotizaciones/presupuestos y contrato de tarifas, órdenes de recogida y control de expediciones."

En ocasiones, las tareas se corresponden con técnicos de comercio exterior en el sentido más restrictivo -o clásico- del término (egresado E), mientras que en otros casos, se observan intersecciones entre las funciones comerciales del área de negocio de la empresa y las funciones propias de los departamentos de comunicación y marketing (egresado F):

Egresado E: "Current responsibilities: Representing XXX XXXX and supporting our partners and customers in SE (mainly in Singapore, Malaysia, Philippines, Indonesia and Sri Lanka) in order to increase brand awareness, 
create a solid and prestigious image, generate sales opportunities and build customer preference towards the brands of our group's portfolio"

Egresado F: “- Comprehensive update of company's website; - Leading role in creation of company web content and industry related expert's opinion document (white paper); - Proofreading and editing of company documents and products presentations and creation of presentations based on a specific field researches; - Creation of matrix of marketing toolsldocuments needed for smooth operation within the commercial departments; - Coordinator in the creative process of sister company's logo and complex rebranding; - Research and analysis on competitors; - Head of marketing assistant in the process of creation of annual activity marketing plan; - Proofreading and updating of the marketing plan; - In-depth research tasks on specific fields; - Cultural liason with Spanish speaking clients; - Facilitation and execution of meetings on various marketing and sales subjects with different representatives of product development, services and financial department; Unification of client related documentation, e.g. Letters (customer testimonials) presentations, user manuals, etc; - Research on upcoming telecom events and selection of suitable ones for company's go-to-market strategy."

El análisis de contenido refleja los siguientes datos respecto a la identificación de las tareas y la identidad profesional de nuestros sujetos:

- 4 sujetos encuestados mencionan expresamente la traducción como tarea o responsabilidad en los puestos que desempeñan. 2 de estos sujetos afirman responsabilizarse (o haberlo hecho) de la traducción de la página web corporativa de su entidad. 1 sujeto menciona la traducción de documentos internos de la empresa.

- Ningún sujeto menciona la interpretación como tarea en su puesto actual.

- 8 de los sujetos encuestados mencionan la traducción entre sus aptitudes (apartado específico que permite el perfil en la red social). Se menciona la traducción (en general), pero también la traducción técnica (en dos perfiles) y la herramienta Trados (en un caso, como aptitud del sujeto). 1 sujeto marca además la Traducción y la Interpretación como aptitudes profesionales de su perfil.

- 4 sujetos mencionan la traducción como tarea desempeñada en cargos similares (empresas internacionales) en su itinerario profesional, pero no en el actual.

Por último, hemos creído conveniente incluir la variable de la formación de estos egresados después de terminar la Licenciatura. En el apartado de formación, a continuación del título de TeI, todos los egresados (con excepción de 2 casos) señalan haber cursado formación de postgrado relacionada 
con la internacionalización de empresas (ítem 3). Los cursos y másteres se repiten con cierta frecuencia, y en el caso de la Escuela de Organización Industrial (EOI) se trata de cursos de formación relativos al programa de becas EXTENDA (Agencia Andaluza de Promoción Exterior). Algunos de los sujetos observados fueron anteriormente becarios del programa de becas de internacionalización EXTENDA (3 de los sujetos) o ICEX (ICEX España Exportación e Inversiones) (2 de los 20). Remitimos a Morón (2012) para conocer la influencia de estas becas sobre la titulación de TeI.

Destacan los siguientes programas, y se recogen a continuación tal y como aparecen redactados en la red (nótese que se repiten los mismos centros de formación con nombre diferente o en lengua inglesa):

- Cámara de Comercio de Sevilla: Expert Degree in International Trade

- Cámara de Comercio de Sevilla: Experto en Dirección de Operaciones Internacionales

- Cámara de Comercio de Tenerife: Curso en transporte y logística internacional (40 horas)

- Cámara de Comercio, Industria y Navegación de Sevilla: Postgraduate, International Business

- EOI - Escuela de Negocios: Postgraduate in International Trade \& Business

- EOI - Escuela de Organización Industrial: Máster Gestión en comercio internacional (414 horas)

- EOI (School of Industrial Organisation): Professional Certificate for Foreign Trade Consultants (Graduate course work)

- IL3- Universidad de Barcelona: Master in International Business and Foreign Trade

- Online Business School, EAE and University of Barcelona: Marketing and Commercial Management, Marketing Management

- UNED: Masters Degree in International Trade, International Business/Trade/Commerce

- Universidad de Valladolid: Master's degree Comercio Exterior

- UPO: Dirección de negocios internacionales

Tabla 3: Formación de postgrado acreditada de los egresados del perfil estudiado

No hemos recabado más información sobre esta formación. Hubiera sido interesante conocer si se trata de formación en línea o presencial, su duración exacta o el lugar de realización. Tampoco hemos cotejado si la formación se produjo antes, durante o después de la primera experiencia profesional en la empresa exportadora.

De forma general, pensamos que la combinación de TeI con un Máster de uno o incluso dos años de formación en administración de empresas o negocios internacionales va a establecerse en los próximos años como uno de los itinerarios más frecuentes para los egresados de TeI en España, y las autoras de este trabajo vienen observando esta tendencia en auge desde la graduación 
de la primera promoción en nuestra universidad en 2008. Sin embargo, como exponemos a continuación, el hecho de haber cursado formación específica en negocios internacionales no parece ser determinante para la autoimagen de los egresados en TeI que, como puede verse en el siguiente apartado, no se ubican a sí mismos en un entorno natural dentro de los puestos anteriormente descritos.

\subsection{Objetivo 2: Autopercepción de los egresados como profesionales de este ámbito}

En los siguientes ejemplos, se refleja la autoconciencia de algunos egresados en TeI de la UPO que están trabajando en empresas españolas (o con sede en España) exportadoras de bienes y servicios. En los casos que presentamos, les habíamos escrito a antiguos alumnos para colaborar con un curso de orientación profesional, y lo que reproducimos aquí son algunas de las respuestas recibidas. Curiosamente, estos egresados pensaron que ellos no eran un buen ejemplo para futuros traductores.

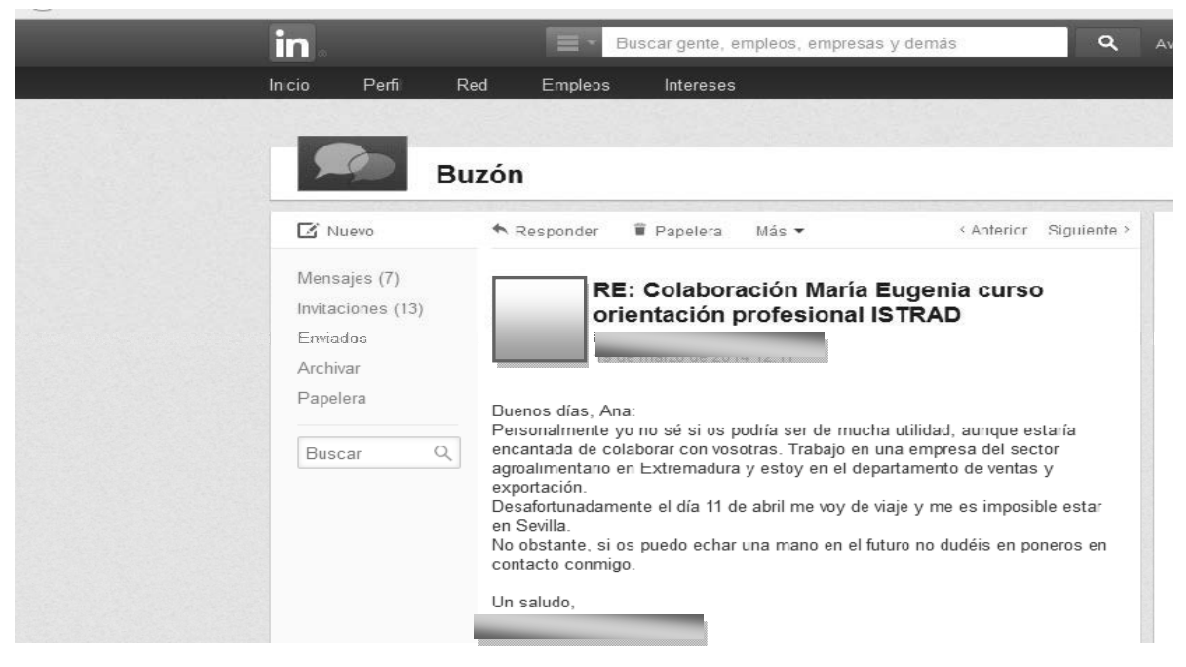

Imagen 1: Primer ejemplo

En el primer ejemplo, la egresada no cree que pueda ser de utilidad en un curso de orientación profesional, porque trabaja en un departamento de ventas y facturación. 


Buzón
$\begin{aligned} & \text { Mensajes (6) } \\ & \text { Invitaciones (13) }\end{aligned}$
Enviados
Archivar
Papelera

Imagen 2: Segundo ejemplo

En el segundo ejemplo, la egresada trabaja en una empresa internacional y se dedica a gestionar la cartera de clientes y la exportación. Ella no observa relación alguna entre la traducción y el comercio exterior.

Hola, Ana!

¿Qué tal estás? ¿Cómo va todo por la UPO?

Pues sigo en Madrid, pero del Máster me gradué en julio y en agosto empecé a trabajar para

) en Comunicación y Afiliación (PR) para el negocio en Alemania y Austria (nonabox.de). Ahora me acaban de hacer un contrato indefinido y he pasado a ser Director of Business Development. El cargo es más grande que yo jaja.

Imagen 3: Tercer ejemplo 
Por su parte, en el tercer ejemplo, la alumna menciona que trabaja para el sector de la comunicación y que mantiene relación con una de las lenguas extranjeras que estudió, si bien su tono discursivo muestra un cierto respeto por el cargo ocupado, como si no fuera un entorno natural para ella.

Como manifiestan estos comentarios, y muy a pesar de los trabajos, cifras y alusiones a la vinculación de la TeI con las relaciones internacionales y el comercio internacional (por otra parte, incorporadas también en la descripción de los documentos de verificación de los títulos de Grado, no en los perfiles específicos que sustentan los títulos, o en los datos de orientación académica de nuestros egresados), ${ }^{9}$ el sentir general de estos licenciados es de no estar dedicándose a aquello para lo que estudiaron. Se ven a sí mismos como intrusos, como alguien que se ha adentrado sin derecho en un ámbito profesional ajeno.

\section{Reflexión en torno al modelo de competencia traductora con objeto de incluir el perfil estudiado}

Tras revisar algunos principios y enfoques sobre la CT, de calado en los estudios de TeI, y una vez observados los puestos ocupados y las funciones desempeñadas por nuestros antiguos alumnos, vamos a aproximarnos nuevamente a la CT para reflexionar sobre qué subcompetencias han sido clave para el éxito de la inserción laboral en el sector de la internacionalización de empresas, y cuáles de ellas pueden reforzarse para mejorar la inserción laboral de los egresados que desean entrar a trabajar en una empresa.

De este modo, y ante la escasa interdisciplinariedad de la oferta educativa española (Calvo 2010: 151), apostamos por un modelo de CT que, en línea con la propuesta de Kelly (2002), sirva como guía para el docente a la hora de afrontar el desarrollo de objetivos de aprendizaje para estudiantes en distintos contextos. Uno de estos contextos sería la CT como competencia transversal en otros estudios universitarios (por ejemplo, para egresados en carreras de economía y empresa, Calvo 2010), habida cuenta de la heterogeneidad del alumnado y sus perfiles de egreso (Anderman y Rogers 2000: 67) y la propia diversidad en cuanto a modelos de formación y cualificación a nivel europeo (Pym, Grin, Sfreddo \& Chan 2013). Así, aunque los modelos de CT conocidos hasta la fecha se centran precisamente en la especificidad de la CT profesional, debemos

9. Véase a modo de ejemplo las págs. 15 o 31 del Libro Blanco del Grado en Traducción e Interpretación de la ANECA (2004), que muestra que el comercio exterior es salida profesional de los egresados consultados (en su estudio y en trabajos previos), si bien este no se incorpora después como perfil específico. 
invertir el proceso de concreción de dicha realidad para que dé cuenta, no de la singularidad de la CT, sino del carácter transversal de dicha competencia en su aplicación a un sector profesional a priori no específico de la Traducción (al menos, no recogido en el Libro Blanco de la TeI en España), como es el del comercio internacional. Ciertamente, las licenciaturas ya habían demostrado que el comercio exterior es una salida recurrente en los estudios de empleabilidad conocidos hasta la fecha en distintas universidades (Aneca 2004, Calvo 2006, Morón 2010, Torres 2012). No obstante, la paradoja de que este campo no quede reflejado en nuestro Libro Blanco nos lleva a preguntarnos el margen curricular o docente con que contamos para atender esta salida profesional en concreto; esto es, hasta qué punto la configuración de los títulos de Grado y la propia docencia de la TeI a este nivel permite una formación orientada a este perfil en la formación de los graduados en TeI. En este contexto, y visto lo anterior, para la definición de nuestro modelo adaptado para este entorno o salida laboral, partimos de la necesidad de redefinir, concretar y reforzar las competencias siguientes:

1. La competencia lingüística. Pese a que algunos estudios han defendido modelos propios en la didáctica de lenguas extranjeras para traductores e intérpretes, pensamos que la competencia lingüística debe ser reforzada en tanto que estos profesionales han de comunicarse de forma oral y escrita y producir textos diversos en distintas lenguas, abarcando un número elevado de situaciones comunicativas del entorno empresarial. Existen actividades elementales en un ambiente empresarial multilingüe que pueden ser entrenadas en un currículo de lengua extranjera si se las incluye, tales como la comunicación al teléfono, la negociación en la compraventa, la comida de negocios, la comunicación con la jerarquía corporativa, etc. En otro orden de cosas, y aunque hemos encontrado entre nuestros egresados gestores de clientes para un único país o región lingüística (por ejemplo, una encargada de la cartera de clientes de Francia y Bélgica o una responsable de las relaciones con Alemania y Austria), el uso del inglés como lengua de los negocios internacionales parece ser predominante en este perfil profesional.

2. La competencia temática. Según Aguayo (2010), el conocimiento temático en materia de comercio internacional era el elemento que mayores reticencias suscitaba en los responsables de comercio internacional consultados en su estudio. Consideraban que un traductor que quisiera integrarse en el mundo de la empresa internacional 
debiera reforzar sus conocimientos en este sentido. También, en el trabajo de Álvarez (2015), los egresados en TeI que trabajan en empresas internacionales revelaban la importancia de la competencia temática (referida al comercio exterior) por encima de cualquier otra en su perfil profesional. La competencia temática siempre ha sido una de las más cuestionadas, pues el traductor ha de desenvolverse con soltura en ámbitos especializados para los que es necesario una formación específica. Es muy frecuente encontrar cursos de especialización realizados en Cámaras de Comercio y otras instituciones privadas en los curricula vitae de estos egresados. Así pues, este campo, convertido en un entorno de trabajo del egresado en TeI es susceptible de ser reforzado en su contenido temático.

3. La competencia intercultural. Puesto que se espera un determinado proceder del especialista en comunicación en contacto con culturas empresariales diversas, sería necesario reforzar el perfil y la conciencia intercultural del sujeto que ha de desenvolverse en este entorno internacional fuertemente condicionado por patrones de comunicación y conducta marcados culturalmente. La propia percepción y estatus de la profesión resulta un particular elemento marcado culturalmente (Pym, Grin, Sfreddo \& Chan 2013) y la formación del estudiante en este sentido puede ser de gran interés, habida cuenta de la demostrada movilidad tras el egreso, no solo de nuestros egresados, sino de los jóvenes españoles, en general.

4. La competencia textual. El egresado trabaja con textos que no son necesariamente objeto de traducción, sino que son redactados directamente en inglés o de forma multilingüe. Además de los encargos de traducción que los estudiantes suelen ver en clase de traducción jurídico-económica, los textos de correspondencia escrita son muy frecuentes, pero también lo son los textos informativos sobre los productos o servicios de la empresa, textos persuasivos sobre la promoción de esos productos, así como documentos mercantiles y financieros. Como afirman Aguayo y Morón (2013) esta competencia ha sido el objetivo fundamental de la mayor parte de los trabajos que han abordado la inclusión de los textos del comercio internacional en el aula de TeI (ya citados en el capítulo introductorio) y esta diversidad sigue apuntando a interesantes vías de investigación.

5. La competencia psicofisiológica. Esta resulta especialmente interesante, dado que el egresado en Traducción ubicado en estos ámbitos de desempeño profesional parece tender a una cierta "pérdida de 
identidad" (Morón, 2010) e incluso a un cierto "sentimiento de intrusismo", tal como hemos apuntado en estas páginas. Paradójicamente, esto ocurre a la vez que siguen emergiendo para los egresados en TeI nuevos perfiles laborales marcados por las nuevas formas de comunicación tecnológicas y globales, como la transcreación, el community management o el marketing electrónico. Al mismo tiempo, este perfil psicofisiológico puede concienciar al sujeto sobre las necesidades y efectos de una buena gestión de la comunicación en la empresa. Así, el traductor integrado en empresas internacionales puede valorar la importancia de la contratación de servicios de traducción para la empresa, puede hacerse él mismo responsable de la revisión de dichos textos y, lo que quizás sea más interesante para la empresa, puede especificar cuáles son las necesidades comunicativas que repercutirán, una vez cubiertas, en una imagen corporativa positiva y en un desarrollo de negocio reforzado.

\section{Conclusiones}

Hemos analizado las actividades de un grupo de traductores que no realizan traducciones en sentido clásico, pero que están desarrollando plenamente las competencias para las que los hemos formado en las universidades, y son precisamente esas competencias las que les permiten desarrollar ágilmente otras habilidades propias de la gestión empresarial, como la capacidad de negociación, o conocimientos y competencias vinculadas con la publicidad o el marketing. Dicho de otra forma: si la CT se redefine completándola con la observación de aquello a lo que se dedican hoy los traductores, abarcará necesariamente el desarrollo de actividades periféricas que nos obligan a entender la formación de traductores en un sentido más amplio.

Nuestros egresados demuestran una clara flexibilidad en su perfil, siendo capaces de acceder a un campo afín, tradicionalmente reservado a otros perfiles académicos más específicos. Su capacidad de adaptación a nuevos entornos de trabajo, su facilidad para familiarizarse con nuevas herramientas informáticas, su entrenamiento para trabajar bajo presión temporal, el hecho de saber trabajar por proyectos y en equipo o sus capacidades de comunicación oral y escrita parecen estar en la base del éxito del perfil profesional analizado, y compensar las carencias temáticas en materia contable o financiera a la hora de su contratación. En cualquier caso, para cubrir estas lagunas, todos los sujetos consultados han buscado programas de formación adecuados para complementar sus conocimientos. No cabe duda de que resulta necesario 
seguir profundizando en la interdisciplinariedad necesaria entre grados y másteres, aún no muy frecuente en nuestro país.

En el contexto actual de crisis económica española y considerando los escandalosos niveles de paro entre los titulados universitarios, no dudamos en defender un modelo curricular enfocado en la empleabilidad y en la formación orientada a cubrir las necesidades del mercado más actuales, poniendo énfasis en la necesidad de unir puentes y de aprovechar las competencias que los estudios de TeI ponen a disposición del mercado, especialmente en el sector de la internacionalización empresarial. Ciertamente, pocas disciplinas han conseguido mantenerse tan al tanto de estos cambios, y han realizado evoluciones tan acompasadas con el ritmo al que este evoluciona.

Hemos mostrado ejemplos reales en nuestro contexto geográfico de cómo las empresas españolas de sectores diferentes al lingüístico están convenciéndose de las ventajas de la contratación de titulados en TeI, aunque dichas contrataciones se hacen con denominaciones de puestos diversos y alejados del término traductor y estos egresados parecen considerar estar dedicándose a "otras cosas ajenas a la traducción". La recurrente cuestión de la identidad del traductor y su visibilidad en el mundo laboral aparece aquí, nuevamente, con interesantes cuestionamientos para futuras investigaciones: ¿siguen siendo traductores los profesionales analizados en estas páginas?, ¿los considera traductores la Traductología?, ¿interesaría a la disciplina que así sea?

Este trabajo, si bien no supera las limitaciones ya esbozadas por Calvo (2006) respecto a los estudios de empleabilidad conocidos, pretende aproximarse a una realidad escasamente visible en nuestro Libro Blanco, que se evidencia en la empleabilidad real registrada por nuestros egresados, así como en la práctica formativa de la traducción especializada.

Por último, en tiempos de críticas frecuentes al Plan Bolonia, pensamos que es interesante destacar las ventajas que trae consigo el desarrollo de competencias transversales, que ha puesto el foco en la empleabilidad, las nuevas tecnologías, la flexibilidad y la movilidad. Queda como asignatura pendiente, no obstante, una mayor interdisciplinariedad en los grados de España, de la que, por otra parte, hemos dado cuenta en esta investigación.

\section{Referencias bibliográficas}

Aguayo Arrabal, Natividad. (2010) Traducción y Comercio exterior ¿Realidad o necesidad? Sevilla: Universidad Pablo de Olavide. Trabajo fin de máster inédito.

Aguayo Arrabal, Natividad \& Cristina Ramírez Delgado (2011) "El traductorintérprete, ¿ ipresente en el comercio exterior?" En: Varios autores. 2011. Actas 
del I Congreso de la Sociedad Española de Lenguas Modernas. Sevilla: Bienza, pp. 11-24.

Aguayo ARRABAL, Natividad \& Marián Morón Martín (2013) "El mercado global para traductores e intérpretes: nuevos objetos y algunos pilares para la investigación futura en traducción-interpretación y comercio internacional.” En: Campos Plaza, Nicolás; Esperanza Alarcón Navio \& Leticia Herrero Rodes (eds.) 2013. Translating Culture- Traduire la Culture- Traducir la cultura. Políticas lingüísticas de comunicación internacional y traducción institucional (Vol. 2). Granada: Comares, pp. 339-354.

Álvarez García, Carmen. (2015) El acceso de los traductores e intérpretes al conocimiento experto en materia económica: especialización en comercio exterior. Sevilla: Universidad Pablo de Olavide de Sevilla. Tesis doctoral inédita.

Anderman, Gunilla \& Margaret Rogers. (2000) "Translator Training between Academia and Profession: A European Perspective." En: Schäffner, Christina $\&$ Beverly Adab (eds.) 2000. Developing Translation Competence. Amsterdam $\&$ Philadelphia: John Benjamins, pp. 63-76.

AnECA. (2004) Título de Grado en Traducción e Interpretación. Madrid: Agencia Nacional de Evaluación de la Calidad y Acreditación. Versión electrónica: <http://www.aneca.es/var/media/150288/libroblanco_traduc_def.pdf>

BARDin, Laurence. (1986) El análisis de contenido. Madrid: Akal.

Calvo Encinas, Elisa. (2006) "Orientación Profesional para Futuros Licenciados de Traducción e Interpretación: Estrategias Centradas en las Necesidades del Estudiantado." En: Bravo Utrera, Sonia \& Rosario García López (eds.) 2006. Estudios de Traducción: Problemas y Perspectivas. Las Palmas de Gran Canaria: Universidad, pp. 633-649.

Calvo Encinas, Elisa. (2010) Análisis curricular de los estudios de traducción e interpretación en España: experiencia curricular del estudiantado. Granada: Universidad. Tesis doctoral inédita.

CALvo ENCINAS, Elisa. (2011) "Translation and/or translator skills as organising principles for curriculum development practice." Jostrans 16, pp. 5-25. Versión electrónica: <http://www.jostrans.org/issue16/art_calvo.pdf>

Centro de Investigaciones Sociológicas, CIS. (2014) Barómetro de febrero 2014. Avance de resultados, Estudio 3013. Versión electrónica: <http://www. rtve.es/contenidos/documentos/cis_barometro_febrero_2014.pdf>

COHEN, Louis \& Lawrence Manion. (1980) Research Methods in Education. Londres: Croom Helm.

ColÁs Bravo, M. Pilar \& Leonor Buendía Eisman. (1998). "La investigación en el campo de la educación." En: Colás Bravo, M. Pilar \& Leonor Buendía Eisman (eds.) Investigación Educativa. Sevilla: Alfar, pp. 176-200.

Comisión EuropeA. (2013) Agenda Digital para Europa. Versión electrónica: $<$ http://ec.europa.eu/digital-agenda/> 
COMISIÓN EUROPEA. (2005) Effects on the European Economy Shortages of Foreign Language Skills in Enterprise. Executive Summary.

CoOK, Thomas D. \& Charles S. Reichardt. (eds.) (1979) Qualitative and Quantitative Methods in Evaluation Research. Beverly Hills, California: Sage Publications.

DELISLE, Jean. (1980) L'analyse du discours comme méthode de traduction: Initiation à la traduction française de textes pragmatiques anglais, théorie et pratique. Ottawa: Presses de l'Université d'Ottawa.

ELAN. (2006) Effects on the European Economy of Shortages of Foreign Language Skills in Enterprise. Versión electrónica: <http://ec.europa.eu/languages/ policy/strategic-framework/documents/elan_en.pdf>

FRIEDMAN, Thomas. (2006). La tierra es plana - Breve historia del mundo globalizado del Siglo XXI. Barcelona: Ediciones Martínez Roca.

GARCíA Ruíz, José Luis \& Carles Manera. (2006) Historia empresarial de España: Un enfoque regional en profundidad. Madrid: Lid Editorial Empresarial.

GILE, Daniel. (2009) Basic Concepts and Models for Interpreter and Translator Training, $2^{\text {a }}$ edición ( $1^{\text {a }}$ edición, 1995). Amsterdam \& Philadelphia: John Benjamins.

Gouadec, Daniel. (2007) Translation as a Profession. Amsterdam \& Philadelphia: John Benjamins.

Hernández SAmpieri, Roberto; Carlos Fernández Collado \& Pilar Baptista Lucio. (2003) Metodología de la investigación, $3^{a}$ edición ( $1^{a}$ edición, 1991). México: McGraw-Hill/Interamericana Editores.

HousE, Juliane. (1981) A Model for Translation Quality Assessment. Tubinga: Gunter Narr.

JimÉnEz CRESPO, Miguel Ángel. (2013) Translation and Web Localization. New York-London: Routledge.

KEARNS, John. (2012) "Curriculum ideologies in translator and interpreter training." En: Hubscher-Davidson, S. \& M. Borodo, 2012. Global trends in translator and interpreter training. London; New York: Continuum, pp. 11-29.

KELlY, Dorothy. (2002) "Un modelo de competencia traductora: bases para el diseño curricular." Puentes: Hacia nuevas investigaciones en la mediación intercultural 1, pp. 9-20.

KIRALY, Donald C. (1995) Pathways to Translation: Pedagogy and Process. Kent: Kent State University Press.

KIRALY, Donald C. (2013) "Towards a View of Translator Competence as an Emergent Phenomenon: Thinking Outside the Box(es) in Translator Education." En: Kiraly, D.; S. Hansen-Schirra, \& K. Maksymski (eds.) New Prospects and Perspectives for Educating Language Mediators. Tübingen: Narr Verlag, pp. 197-224. 
KuZnik, Anna. (2010) El contenido de los puestos de trabajo de los traductores. El caso de los traductores internos en las empresas de traducción de Barcelona. Tesis doctoral inédita. Versión electrónica: <https://ddd.uab.cat/pub/tesis/2011/ hdl_10803_5279/akldel.pdf>

LEE-JAHnKe, Hannelore. (2004) "Qualität - Flexibilität - Effizienz: Die Eckpfeiler einer praxisorientierten Translatorenausbildung." En: Forstner, Martin \& Hannelore Lee-Jahnke (eds.) 2004. Internationales CIUTI-Forum. Marktorientierte Translationsausbildung. Bern: Peter Lang, pp. 59-100.

LÓpez Noguero, Fernando. (2002). "El análisis de contenido como método de investigación." XXI, Revista de Educación 4, pp. 167-179.

MAYORAl ASENSIO, Roberto. (2006) "La traducción comercial." Butlletí de la Associació de Traductors i Intérprets Jurats, diciembre.

Mayoral AsEnsio, Roberto. (2001) "Por una renovación en la formación de traductores e intérpretes: revisión de algunos de los conceptos sobre los que se basa el actual sistema, su estructura y contenidos." Sendebar 12, pp. 311-336.

Medina Reguera, Ana. (2007) "La traducción alemán-español de documentos del comercio exterior: Organización de la tipología textual e integración del contenido temático.” En: Santana, B.; Silvia Roiss \& M. A. Recio (eds.) 2007. Puente entre dos mundos: Últimas tendencias en la investigación traductológica alemán-español. Salamanca: Universidad, pp. 252-260.

Medina ReguerA, Ana. (2015, en prensa): "La investigación en Traducción para el Comercio Internacional: Proyecto COMINTRAD." En: d'Angelis, Antonella, Francisco Núñez-Román \& Estefanía Flores Acuña (eds.): Estudios de traducción de lenguas para fines específicos. Roma: Aracne.

Medina Reguera, Ana \& Cristina Ramírez Delgado. (2015a) "Metodología para evaluar la localización web en España. Primeros resultados aplicados a un corpus de sitios web de pymes agroalimentarias andaluzas.” En: Sánchez Nieto, María Teresa (ed.) 2015. Metodologías y aplicaciones en la investigación en traducción e interpretación con corpus. Valladolid: Universidad, pp. 293-319. Medina Reguera, Ana \& Cristina Ramírez Delgado. (2015b) "La localización de la sección 'productos' en sitios web de empresas exportadoras agroalimentarias." En: Gallego Hernández, Daniel (ed.) 2015. New Insights into Specialised Translation. Monográfico de InTRALinea Online Translation Journal. Versión electronica: <http://www.intralinea.org/specials/article/ la_localizacion_de_la_seccion_productos_en_sitios_web_de_empresas>

Minetur (Ministerio de Industria, Energía y Turismo). (2013) Agenda Digital para España. Versión electrónica: <http://www.agendadigital.gob.es/agendadigital/recursos/Recursos/1.\%20Versión\%20definitiva/Agenda_Digital_para_ Espana.pdf> y <http://www.agendadigital.gob.es/Paginas/Index.aspx>

MORÓN MARTín, Marián. (2012) "La figura del Traductor-Intérprete en procesos de internacionalización: el caso de las convocatorias de becas del ICEX." 
Sendebar 23, pp. 251-274. Versión electrónica: <http://revistaseug.ugr.es/ index.php/sendebar>

MORÓN MARTíN, Marián. (2015) "Traducción y producción lingüística en la comunicación global en entornos empresariales." E-Aesla 1, Instituto Cervantes y Asociación Española de Lingüística Aplicada. Versión electrónica: <http://cvc. cervantes.es/lengua/eaesla/>

Morón MARTín, Marián. (2010) Percepciones sobre el impacto de la movilidad en la formación de traductores: el caso del programa de triple titulación LAE (Lenguas Aplicadas Europa). Granada: Universidad. Versión electrónica. <file:///D:/ Mis\%20documentos/Downloads/18608620.pdf>

MORÓN MARTín, Marián. (2013) "La empleabilidad de egresados en Titulaciones Múltiples y Conjuntas: un caso práctico como balance.” En: Giorgio, Alberti; José Luis Villena \& Martha Beatriz Peluffo (eds.) 2013. Desafíos y oportunidades de la empleabilidad de los egresados universitarios en el contexto internacional. Madrid: McGraw-Hill, pp. 29-52.

Navarro Arancegui, Mikel. (2007) El entorno económico y la competitividad en España. Bilbao: Universidad de Deusto.

NoRD, Christiane. (1991) Text Analysis in Translation: Theory, Methodology and Didactic Application of a Model for Translation-Oriented Analysis. Amsterdam: Rodopi.

OlOHAN, Maeve. (2007) "Economic Trends and Developments in the Translation Industry. What Relevance for Translator Training?" The Interpreter and Translator 1:1, pp. 37-63.

PACTE (2009) "Results of the validation of the PACTE Translation Competence model: Acceptability and decision-making." Across Languages and Cultures 10:2, pp. 207-230.

PACTE (2014) "First results of PACTE group's experimental research on translation competence acquisition: The acquisition of declarative knowledge of translation." En: Muñoz Martín, Ricardo (ed.) Minding Translation - MonTI Special Issue 1, pp. 85-115.

PyM, Anthony. (2003) "Redefining Translation Competence in an Electronic Age: In Defence of a Minimalist Approach.” Meta 48:4, pp. 481-497.

PYM, Anthony; François Grin; Claudio Sfreddo \& Andy L. J. Chan. (2013) The status of the translation profession in the European Union. New York: Anthem Press.

Rico, Celia. (n.d.) "Formación continua para el traductor profesional. Campos especializados y Nuevas tecnologías." Versión electrónica: <http://www.slideshare.net/celiarico/traduccion-profesional-6812370>

RIKE, Sissel Marie. (2013) "Bilingual corporate websites - from translation to transcreation?" Jostrans 20, pp. 68-85. 
RoBinson, Douglas. (1997, 2003) Becoming a Translator: An Introduction to the Theory and Practice of Translation. London; New York: Routledge.

Romaine, Mathew \& Jennifer Richardson. (2009) State of the Translation Industry 2009: Smarter, more casual. MyGengo. Inc.

RomÁn Mínguez, Verónica. (2008) "La traducción (inglés-español) de los documentos negociales: reflexiones sobre didáctica y competencia traductora." En: Ortega Arjonilla, Emilio (ed.) 2008. La traducción e interpretación jurídicas en la UE. Retos para la Europa de los ciudadanos. Granada: Comares, pp. 113-132.

Román MíngueZ, Verónica. (2012) "Acquiring Competencies in EconomicFinancial Translation: from the Classroom to the Marketplace." Revista Alicantina de Estudios Ingleses 25, pp. 387-400.

SALES, Dora. (2004) Puentes sobre el mundo: cultura, traducción y forma literaria en las narrativas de transculturación de José María Arguedas y Vikram Chandra. Berna: Peter Lang.

SCHÄFFnER, Christina. (2012) "Translation Competence: Training for the Real World.” En: Hubscher-Davidson, Séverine \& Michal Borodo (eds.) 2012. Global trends in translator and interpreter training. London: Continuum, pp. 30-44.

Socorro Trujillo, Karina. (2008) Aspectos textuales y terminológicos de documentos mercantiles del comercio internacional: herramientas aplicables a la formación de traductores. Las Palmas de Gran Canaria: Servicio de publicaciones de la ULPGC.

SOCORRO Trujillo, Karina. (2012) "La enseñanza de la traducción directa de la correspondencia comercial inglés-español: hacia una competencia textual del comercio internacional." Sendebar 23, pp. 301-320.

SuAU JimÉNEZ, Francisca. (2010) La traducción especializada en inglés y español en géneros de Economía y Empresa. Madrid: Arco Libros.

TORRES-Hostench, Olga. (2012). "Occupational Integration Training in Translation." Meta 57:3, pp. 787-811.

UlRYCH, Margherita. (2005) "Training Translators: programmes, curricula, practices." En: Tennent, Martha (ed.) 2005. Training for the New Millennium: Pedagogies for Translation and Interpreting. Amsterdam; Philadelphia: John Benjamins.

WAY, Catherine. (2008) "Systematic assessment of translator competence: in search of Achilles' heel." En: Kearns, John (ed.) 2008. Translator and interpreter training. London: Continuum, pp. 88-103.

WILSS, Wolfram. (1976) "Perspectives and Limitations of a Didactic Framework for the Teaching of Translation." En: Anderson; R. Bruce W. \& Richard W. Brislin (eds.) 1976. Translation: Applications and Research. New York: Gardner Press, pp. 117-137. 


\section{NOTAS BIOGRÁFICAS / BIONOTES}

MARIÁN MORÓN MARTín es doctora en Traducción e Interpretación por la Universidad de Granada. Docente en esta universidad, donde forma parte del grupo AVANTI desde el año 2003, y luego en la Universidad Pablo de Olavide desde el año 2006. Sus líneas de investigación se centran en la traducción especializada y, más concretamente, en la traducción que se desempeña en entornos empresariales internacionales y en y para el comercio exterior. Es docente del Máster Oficial de Enseñanza Bilingüe y del MO de Comunicación Internacional, Traducción e Interpretación de la UPO, y miembro del proyecto de I+D+i COMINTRAD.

MARIÁN MORÓN MARTín holds a doctorate in translation and interpreting from the University of Granada. She taught at the University of Granada, where she was part of the AVANTI group, starting in 2003, and has been teaching at Universidad Pablo de Olavide since 2006. Her research interests focus on specialized translation and, more specifically, on translation serving international business environments and in and for foreign trade. She teaches in the Official Master of Bilingual Education and the O.M. of International Communication, Translation and Interpretation at UPO. She is also a member of the COMINTRAD R\&D\&I project.

Ana Medina Reguera es profesora del Dpto. de Filología y Traducción de la Universidad Pablo de Olavide de Sevilla desde 2004. Doctora en Filología Alemana, sus líneas de investigación son la traducción de lenguas de especialidad alemán-español, la traducción para el comercio exterior, la didáctica de lenguas con fines profesionales y el análisis del discurso administrativo y empresarial. Es docente de varios másteres oficiales y propios de la UPO y la UIMP. En la actualidad dirige el Proyecto COMINTRAD "Comercio Internacional y Traduccion: Localizacion de webs corporativas y E-Marketing Multilingüe para fomentar la internacionalizacion de las pymes españolas".

Ana Medina Reguera teaches in the Department of Languages and Translation at Universidad Pablo de Olavide since 2004. She holds a doctorate in German Studies. Her research interests include German-Spanish specialized translation, foreign trade translation, language teaching for professional purposes and analysis of administrative and business discourse. She teaches in several official master's degree programs and specific master's degree programs at UPO and UIMP. She is currently head of the R\&D\&I project COMINTRAD "International Trade and Translation: Localization of Corporate Websites and Multilingual e-Marketing to Promote the Internationalization of Spanish SMEs". 